\title{
Rhabdomyosarcoma of the left atrium of the heart in a child - case report
}

\author{
Konrad Stępień ${ }^{1}$, Małgorzata Czogała²,3, Katarzyna Garus ${ }^{3}$, Łukasz Wyrobek4, Elżbieta Wójcik5, \\ Janusz Skalski ${ }^{5,6}$, Walentyna Balwierz ${ }^{2,3}$ \\ 'Student Scientific Group of Paediatric Oncology and Haematology, Institute of Paediatrics, Jagiellonian University \\ Medical College, Krakow, Poland \\ 2Department of Oncology and Haematology, University Children's Hospital, Krakow, Poland \\ ${ }^{3}$ Department of Paediatric Oncology and Haematology, Institute of Paediatrics, Jagiellonian University Medical College, \\ Krakow, Poland \\ ${ }^{4}$ Department of Radiology, University Children's Hospital, Krakow, Poland \\ ${ }^{5}$ Department of Cardiosurgery, University Children's Hospital, Krakow, Poland \\ ${ }^{6}$ Department of Paediatric Cardiosurgery, Institute of Paediatrics, Jagiellonian University Medical College, Krakow, Poland
}

\section{ABSTRACT}

Primary tumours of the heart are rare in children. Most of them are benign. Sarcomas predominate among malignant tumours and are characterised by disadvantageous prognosis, especially in the presence of metastases. We present a case of a 16-year-old boy admitted to the hospital with signs of worsening exercise tolerance and suspected left atrial myxoma. The patient was urgently treated with cardiac surgery. Only partial tumour resection was possible. Rhabdomyosarcoma (RMS) embryonale was diagnosed. Stage IV of the disease was established (metastases to the left lung). A good initial response to chemotherapy was observed. In control studies, during the maintenance chemotherapy (15 months from the diagnosis of RMS) progression of the disease (PD) was found: right forearm tumour with metastasis near the left adrenal gland. Despite the intensification of treatment, there was resistance to successive lines of chemotherapy. The patient died of the disease progression.

\section{KEY WORDS:}

children, treatment, heart, malignant neoplasm.

\section{INTRODUCTION}

Primary cardiac tumours are rarely diagnosed in the paediatric population. They represent about $0.027-0.32 \%$ of all childhood neoplasms [1]. The most common of these (90\%) are benign tumours. Sarcomas, including rhabdomyosarcoma (RMS), as well as extremely rare cases of fibrosarcoma, synovial sarcoma, and granulocytic sarcoma [2], dominate among malignant heart tumours [3].

Moreover, until now only single cases of secondary heart involvement by neuroblastoma, leukaemia, lympho- ma, and melanoma have been reported [4]. Prognosis in sarcomas is still disadvantageous, especially in the presence of metastases. In stage IV the five-year survival rate is about $20-30 \%$ [5]. Only a few cases of disseminated form of RMS of the heart have already been described.

\section{CASE REPORT}

We present a case of a 16-year-old boy admitted with suspicion of left atrial myxoma with signs of gradually worsening physical exertion tolerance for three months.

\section{ADDRESS FOR CORRESPONDENCE:}

Walentyna Balwierz, Department of Paediatric Oncology and Haematology, Institute of Paediatrics, Jagiellonian University Medical College, 265 Wielicka St., 30-663 Krakow, Poland,

e-mail: onkhemdz@cm-uj.krakow.pl,walentyna@balwierz.com,walentyna.balwierz@uj.edu.pl 


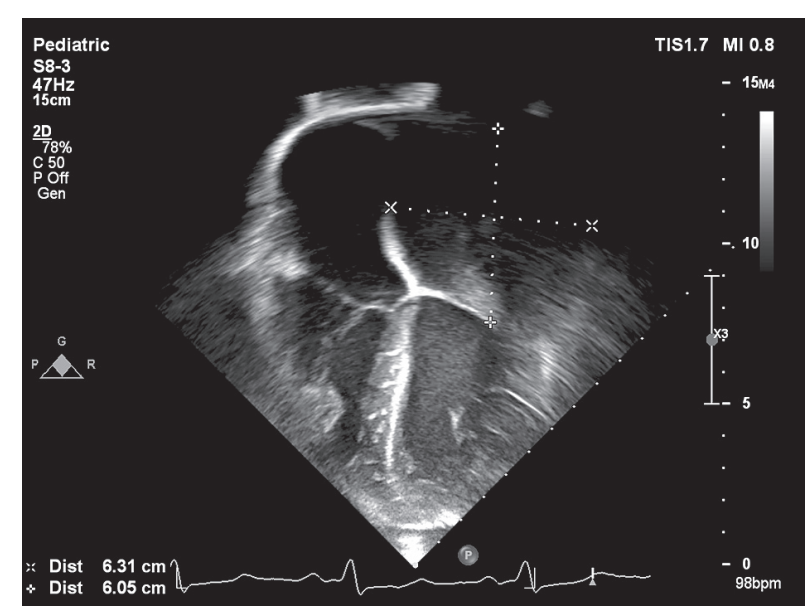

FIGURE 1. Tumour of the left atrium with dimensions $6.3 \times 6.1 \times 4 \mathrm{~cm}$ visualised with transthoracic echocardiography

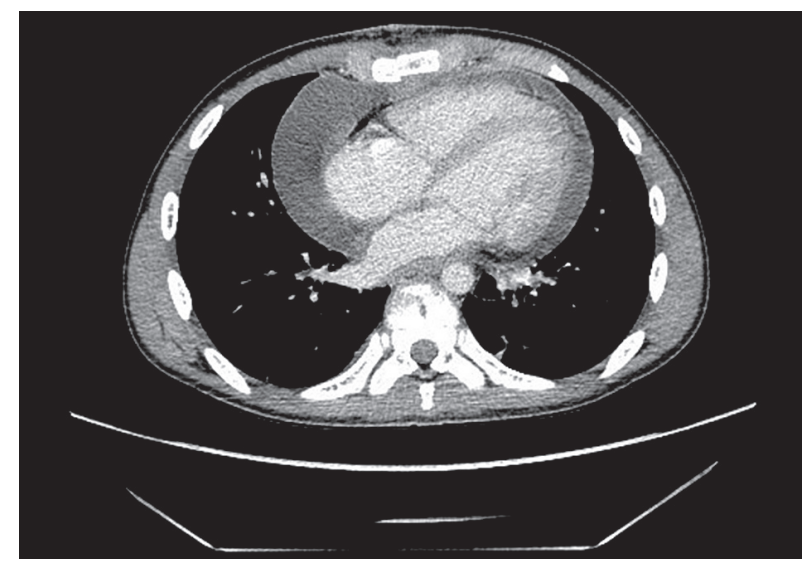

FIGURE 3. Postoperative changes in the heart in chest computed tomography scan

There was systolic murmur over the apex of the heart in physical examination. No other abnormalities were found. Due to the increasing symptoms of heart failure, standard laboratory tests, electrocardiography (ECG), and transthoracic echocardiography (TTE) were performed. ECG revealed the features of enlargement of the left atrium. In TTE a $6.3 \times 6.1 \times 4 \mathrm{~cm}$ tumour attached to the posterior lateral wall of the left atrium was visualised (Fig. 1). The tumour narrowed the mitral valve and the left ventricular outflow tract (Fig. 2). Based on the TTE image, left atrial myxoma was suspected. The patient was urgently qualified for cardiac surgery.

The tumour filling the left atrium of the heart was visualised intraoperatively. The main tumour mass was removed, dissecting it from the posterior flap of the mitral valve. The tumour infiltrated the posterior wall of the left atrium. Complete resection was not possible. Postoperative transoesophageal echocardiography (TEE) visualised acceptable mitral valve regurgitation (I/II grade). Histopathological examination revealed RMS embryonale anaplastic variant (vimentin+, desmin+, myogenin+).

Computed tomography (CT) performed seven days after surgery showed: irregular masses in the left-poste-

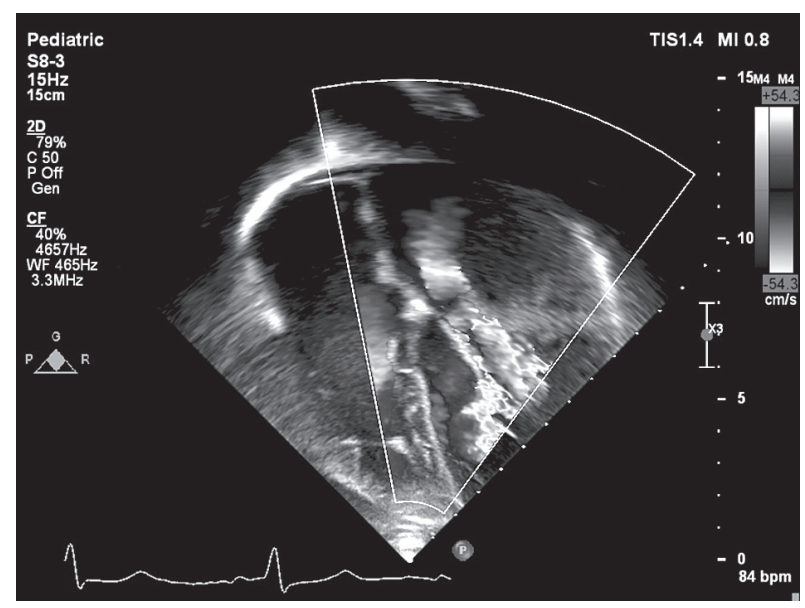

FIGURE 2. Narrowing of the mitral valve by the left atrial tumour visualised by transthoracic echocardiography with colour Doppler function

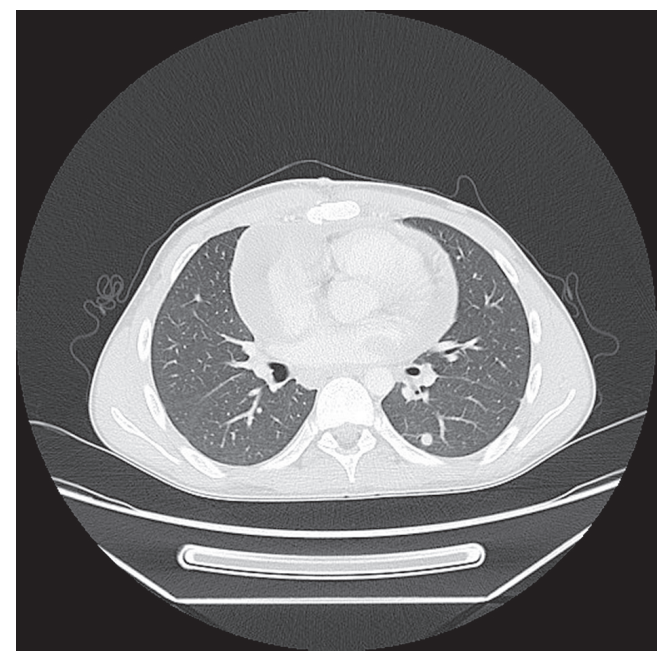

FIGURE 4. Metastatic change in the sixth left lung segment visualised in chest computed tomography scan

rior part of the left atrium and a large amount of dense fluid in the pericardium corresponding to postoperative changes (Fig. 3), change in the sixth left lung segment (6 $\mathrm{mm}$ diameter) (Fig. 4), and thickened left adrenal gland with a nodule of $9 \mathrm{~mm}$ diameter. There were no abnormalities in bone scintigraphy or in the studies evaluating bone marrow (biopsy and trepanobiopsy).

Stage IV was determined. The CWS 2006 protocol for the high-risk group was initiated [6]. In a control CT scan performed in the ninth week of treatment, a reduction of the left lung lesion to $3.5 \mathrm{~mm}$ was observed. The change in the adrenal gland remained stable. According to the protocol, during the course of chemotherapy, the tumour site and the metastatic lesion in the left lung were irradiated (4140 Gy in 23 fractions) with a $6.15 \mathrm{MV}$ photon beam from linear accelerator. In the next CT scan (eighth month of treatment) there was no previously described lung lesion, but a new small change $(3 \mathrm{~mm}$ ) was found. A nodule in the left adrenal gland was unchanged and was considered as independent of the sarcoma. During treatment 
several complications were observed, such as: leukopaenia (WHO grade IV), anaemia (WHO grade III), oral mucositis, and lower left limb polyneuropathy. Cardiac complications (sinus tachycardia and diastolic dysfunction of left ventricle) developed in the sixth month of treatment. In a control physical examination (15 months after diagnosis of RMS) during maintenance chemotherapy with OTI/ OTE scheme [6] a tumour was found in the proximal part of the right forearm. Computed tomography revealed soft tissue tumour in the right forearm $(5.2 \times 4.2 \times 9 \mathrm{~cm})$ and a new nodular change near the left adrenal gland independently of the previously described small stable tumour. Disease progression was confirmed by histopathological examination of the specimen from the tumour of the arm. Despite the intensification of treatment (second and subsequent lines of therapy), further progression was observed. The patient died 12 months after disease progression, 27 months after diagnosis.

\section{DISCUSSION}

The presented case refers to a difficult clinical situation - a primary, incompletely removed RMS located in the heart of a child, with distant metastasis to the lung. Primary cardiac tumours, especially malignant, are extremely rare in the paediatric population $[1,3]$. The description of this case can be used to analyse several important problems that appear during the diagnosis and treatment of this type of patient.

Histopathological examination revealed RMS embryonale anaplastic variant. Two main histological subtypes of RMS are distinguished: embryonal and alveolar. More favourable prognosis for the embryonal subtype has been shown [7], but anaplasia, found in $13 \%$ of patients with RMS embryonale, significantly worsens the results of treatment [8].

Before the cardiac surgery, the boy underwent the necessary set of tests enabling proper qualification. In recent years, non-invasive methods of intracardiac tumour diagnostics have been extensively studied. They focus mainly on the assessment of tumour structure, its mobility, attachment to the heart wall, and its blood supply [9, 10]. The primary aim is to differentiate between benign lesions (myxomas and intracardiac clots) and malignant tumours - in the paediatric population most often RMS. Early diagnosis makes it possible to choose the appropriate treatment methods. However, despite this, the final diagnosis can only be made on the basis of histopathological examination. The difference between the interpretation of echocardiography and the result of postoperative material analysis in this case confirms the effectiveness of the guidelines.

Accurate imaging also plays an important role in a patient's qualification for appropriate adjuvant treatment. In the patient's chest CT, a metastatic lesion in the left lung was detected, and the highest stage IV was determined.
Proper diagnostic imaging also has proven prognostic value. Using general information about the patient and the results of imaging tests, assessment using the Oberlin risk criteria can be made [11]. Based on these criteria, the expected three-year event-free survival (EFS) was only $20 \%$. Baseline prognosis in our patient was highly unfavourable.

Currently, the standard treatment in patients with primary disseminated RMS is combined therapy with surgical methods, radiotherapy, and chemotherapy [5]. In the above case the CWS 2006 protocol dedicated to paediatric patients with high-risk RMS was applied. In recent years a frequently discussed topic has been the strategy of dealing with isolated metastatic changes in the lungs. This problem is clinically important. The lungs are the most common location of metastatic lesions in patients with RMS. An important document that raises this issue is the report of the CWS study group [12]. The use of local treatment methods - radiotherapy and surgical procedures - is not associated with improved prognosis. In the presented case irradiation of both metastatic changes in the left lung and residual masses in the left atrium of the heart was performed, which was associated with cardiac complications observed in the sixth month of treatment - sinus tachycardia and diastolic dysfunction of the left ventricle. This procedure was motivated by the initially unfavourable prognosis in this patient. The paediatric population is particularly at risk for cardiovascular complications during radiotherapy involving the chest region. Radiation-related heart disease can affect every heart structure and is mainly related to diffuse myocardial dysfunction (observed in this case), pericarditis and pericardial fibrosis, premature development of coronary artery disease, and degeneration of the heart valves [13]. The early development of cardiac complications in this case is probably caused by the high intensity of treatment as well as the synergistic cardiotoxic effect of radiotherapy and the anthracyclines in the CWS 2006 protocol [14].

The occurrence of PD, after 15 months of intensive combined therapy, confirms that patients with disseminated RMS are characterised by a very unfavourable prognosis after using standard methods of treatment. Despite the efforts of many research groups, the results of the treatment have not yet been significantly improved. Therefore, it seems necessary to constantly develop local and systemic methods of treatment.

In the above case, only partial resection of the primary tumour was possible. A novel method of the treatment, described in the literature, ensuring complete resection, is heart transplantation $[15,16]$. New radiotherapeutic techniques, such as intensity-modulated radiation therapy (IMRT), proton therapy, and brachytherapy, are continuing to improve treatment results by increasing the radiation dose delivered to the tumour site with less radiation of surrounding tissues [17]. It will certainly improve the results of treatment of non-diffuse disease. 
TABLE 1. Recently published research concerning the use of targeted therapy in rhabdomyosarcoma in children

\begin{tabular}{|l|c|c|c|c|}
\hline Author of the study & Year of publication & Name of the drug & Targeted pathway & Research stage \\
\hline Fouladi M et al. [19] & 2010 & vorinostat & histone deacetylases (HDAC) & First phase \\
\hline Mascarenhas L et al. [20] & 2014 & temsirolimus vs. bevacizumab & mTOR kinase vs. VEGF & Second phase \\
\hline Kim A et al. [21] & 2015 & sorafenib & $\begin{array}{c}\text { C-RAF, B-RAF, VEGFR-2,-3, } \\
\text { PDGFR- } \beta, \text { FLT3, c-KIT }\end{array}$ & Second phase \\
\hline Lee A et al. [22] & 2015 & pazopanib & $\begin{array}{c}\text { VEGFR-1,-2,-3, PDGFR-a,- } \beta, \\
\text { FGFR, c-KIT }\end{array}$ & Second phase \\
\hline Chisholm JC et al. [23] & 2017 & bevacizumab & VEGF & Second phase \\
\hline Glod J et al. [24] & 2018 & cediranib & VEGFR-1,-2,-3 & Second phase \\
\hline
\end{tabular}

In recent years, despite numerous studies investigating the modification of chemotherapy, using maintenance chemotherapy and myeloablative therapy, no significant improvement in the treatment outcomes was observed in either paediatric or adult patients. Therefore, particularly high expectations in the future are associated with the introduction of targeted therapies, using numerous abnormalities in intracellular pathways found in RMS [18]. The most important and recent reports concerning the use of this class of drugs in RMS are presented in Table 1. Perhaps their use will lead to improved prognosis in this group of patients.

\section{CONCLUSIONS}

The presented case description raises a difficult clinical problem. In a patient with disseminated RMS embryonale anaplastic variant, with an ultra-rare primary tumour location, despite the use of combined treatment, only a shortterm partial remission of the disease was obtained. Due to the progression of the disease and resistance to successive lines of chemotherapy, the patient died. Undoubtedly, new and innovative methods of treatment are needed to improve survival in this specific group of patients.

\section{DISCLOSURE}

The authors declare no conflict of interest.

\section{REFERENCES}

1. Gunther T, Schreiber C, Noebauer C, et al. Treatment strategies for pediatric patients with primary cardiac and pericardial tumors: a 30-year review. Pediatr Cardiol 2008; 29: 1071-1076.

2. Schaffer LR, Caltharp SA, Milla SS, et al. Rare presentation of four primary pediatric cardiac tumors. Cardiovasc Pathol 2016; 25: 72-77.

3. Monge MC, Walterhouse DO, Kalapurakal JA, et al. Resection of Intrapericardial Rhabdomyosarcoma in a Child. World J Pediatr Congenit Heart Surg 2016; 7: 108-111.

4. Uzun O, Wilson DG, Vujanic GM, et al. Cardiac tumours in children. Orphanet J Rare Dis 2007; 2: 11.

5. Egas-Bejar D, Huh WW. Rhabdomyosarcoma in adolescent and young adult patients: current perspectives. Adolesc Health Med Ther 2014; 5: 115-125.
6. Kazanowska B, Węcławek-Topol J. Mięsaki tkanek miękkich. In: Zalecenia postępowania diagnostyczno-terapeutycznego w nowotworach złośliwych - 2013 r. Tom 3, Krzakowski M, Warzocha K. Via Medica, Gdańsk 2014: 1124-1149.

7. Meza JL, Anderson J, Pappo AS, et al. Analysis of prognostic factors in patients with nonmetastatic rhabdomyosarcoma treated on intergroup rhabdomyosarcoma studies III and IV: the Children's Oncology Group. J Clin Oncol 2006; 24: 3844-3851.

8. Qualman S, Lynch J, Bridge J, et al. Prevalence and clinical impact of anaplasia in childhood rhabdomyosarcoma: a report from the Soft Tissue Sarcoma Committee of the Children's Oncology Group. Cancer 2008; 113: 3242-3247.

9. Ma J, Sun JP, Chen M, et al. Left Atrial Rhabdomyosarcoma. Circulation 2014; 129: e503-e505.

10. Kirkpatrick JN, Wong T, Bednarz JE, et al. Differential diagnosis of cardiac masses using contrast echocardiographic perfusion imaging. J Am Coll Cardiol 2004; 43: 1412-1419.

11. Oberlin O, Rey A, Lyden E, et al. Prognostic factors in metastatic rhabdomyosarcomas: results of a pooled analysis from United States and European cooperative groups. J Clin Oncol 2008; 26: 2384-2389.

12. Dantonello TM, Winkler P, Boelling T, et al. Embryonal rhabdomyosarcoma with metastases confined to the lungs: report from the CWS Study Group. Pediatr Blood Cancer 2011; 56: 725-732.

13. Schultz-Hector S, Trott KR. Radiation-induced cardiovascular diseases: Is the epidemiologic evidence compatible with the radiobiologic data? Int J Radiat Oncol Biol Phys 2007; 67: 10-18.

14. Basavaraju SR, Easterly CE. Pathophysiological effects of radiation on atherosclerosis development and progression, and the incidence of cardiovascular complications. Med Phys 2002; 29: 2391-2403.

15. Grandmougin D, Fayad G, Decoene C, et al. Total orthotopic heart transplantation for primary cardiac rhabdomyosarcoma: factors influencing long-term survival. Ann Thorac Surg 2001; 71: 1438-1441.

16. Selman AR, Ubilla SM, Espinoza HJ, et al. Heart autotransplantation for the treatment of a rhabdomyosarcoma of the left ventricle. Report of one case. Rev Med Chil 2012; 140: 775-779.

17. Yang JC, Wexler LH, Meyers PA, et al. Intensity-modulated radiation therapy with dose-painting for pediatric sarcomas with pulmonary metastases. Pediatr Blood Cancer 2013; 60: 1616-1620.

18. Huh WW, Skapek SX. Childhood rhabdomyosarcoma: new insight on biology and treatment. Curr Oncol Rep 2010; 12: 402-410.

19. Fouladi M, Park JR, Stewart CF, et al. Pediatric Phase I Trial and Pharmacokinetic Study of Vorinostat: A Children's Oncology Group Phase I Consortium Report. J Clin Oncol 2010; 28: 3623-3629.

20. Mascarenhas L, Meyer WH, Lyden E, et al. Randomized phase II trial of bevacizumab and temsirolimus in combination with vinorelbine (V) and cyclophosphamide (C) for first relapse/ disease progression of rhabdomyosarcoma (RMS): a report from the Children's Oncology Group (COG). J Clin Oncol 2014; 32 (Suppl): 10003-10003. 
21. Kim A, Widemann BC, Krailo M, et al. Phase 2 trial of Sorafenib in Children and Young Adults with Refractory Solid Tumors: A Report from the Children's Oncology Group. Pediatr Blood Cancer 2015; 62: $1562-1566$.

22. Lee A, Bender JG, Weigel B, et al. A phase II study of pazopanib in children, adolescents, and young adults with refractory solid tumors. J Clin Oncol 2015; 33 [ahead of print]. DOI: 10.1200/jco.2015.33.15_ suppl.tps10081.

23. Chisholm JC, Merks JHM, Casanova M, et al. Open-label, multicentre, randomised, phase II study of the EpSSG and the ITCC evaluating the addition of bevacizumab to chemotherapy in childhood and adolescent patients with metastatic soft tissue sarcoma (the BERNIE study). Eur J Cancer 2017; 83: 177-184.

24. Glod J, Cohen JW, Widemann BC, et al. Cediranib phase II study in children with metastatic alveolar soft part sarcoma (ASPS). J Clin Oncol 2018; 36 (Suppl): 10540-10540. 